\title{
Fractional Powers of Operators of Tsallis Ensemble and their Parameter Differentiation
}

\author{
A. K. Rajagopal \\ Naval Research Laboratory, \\ Washington D. C. 20375-5320, USA \\ Received 07 December, 1998
}

\begin{abstract}
We develop four identities concerning parameter differentiation of fractional powers of operators appearing in the Tsallis ensembles of quantum statistical mechanics of nonextensive systems. In the appropriate limit these reduce to the corresponding differentiation identities of exponential operators of the Gibbs ensembles of extensive systems derived by Wilcox.
\end{abstract}

\section{Introduction}

Wilcox [1] in 1967 published a seminal paper entitled

"Exponential Operators and Parameter Differentiation in Quantum Physics". This paper was centered around the following remarkable identity:

If $\hat{H}(\lambda)$ is an operator depending on a parameter $\lambda$, then

$$
\frac{\partial}{\partial \lambda} \hat{Q}(\lambda, \beta)=-\int_{0}^{\beta} d u \hat{Q}(\lambda, \beta) \hat{Q}^{-1}(\lambda, u) \frac{\partial \hat{H}(\lambda)}{\partial \lambda} \hat{Q}(\lambda, u)
$$

where

$$
\hat{Q}(\lambda, u)=\exp -u \hat{H}(\lambda)
$$

From this he went on to obtain several other identities in elegant ways which are all central in the development of quantum time evolution, Gibbsian ensembles in equilibrium quantum statistical mechanics, perturbation expansions, inequalities concerning correlation functions etc., all of which depend on the appearance of the exponential operator of the form introduced in Eq.(1). For a comprehensive account of the ramifications of this identity, see Appendix A in Vol. I of Grandy's Textbook on Statistical Mechanics [2]. In Appendix D of the Vol.II of his text, he uses this identity to establish inequalities of various covariance functions which are just the quantum mechanical variances and covariances of quantities of interest. It is useful to recall that the exponential form in the Gibbsian ensem- ble arises from the principle of maximum von Neumann entropy, $S_{1}=-\operatorname{Tr} \hat{\rho} \ln \hat{\rho}$, with $\operatorname{Tr} \hat{\rho}=1$, subject to the given mean energy of the system, $E=\operatorname{Tr} \hat{\rho} \hat{H}$, as explained in [2], for example.

In 1988, Tsallis [3] introduced a different ensemble for describing a large variety of nonextensive systems for which the exponential form of the operator is replaced by a monomial fractional power of the form

$$
\hat{Q}_{T}(\lambda, \beta)=[1-(1-q) \beta \hat{H}(\lambda)]^{q /(1-q)},
$$

where q specifies the system nonextensivity. Nonextensivity comes about in systems with long-range interactions between constituent particles of the system. This goes over to the Gibbsian form given in Eq.(1) when $q$ is set equal to unity, which is appropriate whenever 
the system consists of particles which are either noninteracting or interacting with short-range forces. The operator in Eq.(2) replaces the exponential operator in Eq.(1) when statistical expectation values of quantities of interest are to be calculated in the Tsallis formalism. Here the Tsallis entropy $S_{q}=-\operatorname{Tr}\left(\hat{\rho}-\hat{\rho}^{q}\right) /(1-q)$, is maximized subject to the given q-expectation value (a different form of the q-expectation value to be defined later is sometimes preferred) of the Hamiltonian, $E_{q}=\operatorname{Tr} \hat{\rho}^{q} \hat{H}$, besides the usual normalization, $\operatorname{Tr} \hat{\rho}=1$. It is in this form (as well as the different form alluded to above) that the formal structure of Statistical Mechanics is preserved namely - Legendre transform nature of the free energy. This ensemble has now been used to develop [4] a Green function theory of manyparticle nonextensive systems in much the same way as the Gibbsian ensemble is used in the Green function theory of the corresponding extensive systems. With this development, the program of statistical mechanics of nonextensive systems is achieved in a manner that is formally complete in parallel to the conventional statistical mechanics of extensive systems.

It may be important to give the reader some compelling reasons for setting up another ensemble different from the traditional Boltzmann-Gibbs ensemble for dealing with nonextensive systems. We first recall that among the statistical distributions, the exponential - class played very important role in the analysis of many phenomena. (see E. T. Jaynes [5] for a discussion of these aspects). These can all be derived from a maximum entropy principle subject to some constraints in which the entropy functional is chosen to be the Gibbs-von Neumann form, $S_{1}=-\operatorname{Tr} \hat{\rho} \ln \hat{\rho}$. There are many other probability distributions possessing long tails such as Pareto, Levy, etc. which are of the monomial - class, not related to the exponential class. These are not derivable from the maximum entropy principle with the Gibbs-von Neumann form for the entropy functional. These cover many phenomena which do not come under the rubric of "extensive" class of systems which were traditionally treated in physical and other sciences. It is this important gap that the Tsallis ensembles cover. As far as we are aware, there is no mathematical or physical argument to rule out the applicability of the Tsallis ensemble nor do we know any demonstration that the exponential-class covers every conceivable situation in physical and other sciences so that the universality of the Boltzmann-Gibbs ensemble may be considered as the only one paramount form. In discussing sensitivity to initial conditions of nonlinear dynamical systems, similar "exponential" and "power" law sensitivities have been discussed recently $[6,7]$ in the context of the use of von Neumann (Kolmogorov and Sinai) and Tsallis entropies in their quantification. The Tsallis ensemble with $q \neq 1$ deals with Hamiltonians of systems with long-range interactions which may exhibit nontrivial anomalies in their ergodicity and mixing properties. For systems which are noninteracting or interacting systems with short-range forces, one certainly has $q=1$ (Boltzman-Gibbs class).

\section{Four theorems}

In this paper, we first obtain the counterpart of the Wilcox theorem, Eq.(1), for the Tsallis form of the operator, and then deduce three others. It is not out of place here to mention that some of these have been recently stated without derivation [8] in developing the dynamic linear response of a nonextensive system based on Tsallis framework. It is worth pointing out that every one of these theorems has its counterpart in the exponential version, derived by Kubo, Karplus and Schwinger in different physical situations of the respective authors' interests. Wilcox's theorem unifies all of these in a single, elegant form, from which all others follow.

\section{THEOREM I:}

$$
\frac{\partial}{\partial \lambda} \hat{Q}_{T}(\lambda, \beta)=-q \int_{0}^{\beta} d u \hat{Q}_{T}(\lambda, \beta) \hat{Q}_{T}^{-1}(\lambda, u) \frac{\partial \hat{\tilde{H}}(\lambda, u)}{\partial \lambda} \hat{Q}_{T}(\lambda, u)
$$




$$
\text { where } \frac{\partial \hat{\tilde{H}}(\lambda, u)}{\partial \lambda}=(1-u(1-q) \hat{H}(\lambda))^{-1} \frac{\partial \hat{H}(\lambda)}{\partial \lambda}(1-u(1-q) \hat{H}(\lambda))^{-1}
$$

Prof: Let

$$
\hat{F}_{T}(\lambda, \beta)=\frac{\partial \hat{Q}_{T}(\lambda, \beta)}{\partial \lambda} .
$$

Then differentiating with respect to $\beta$, interchanging the order of differentiation, using the definition (4), and using the definition (3) given above, we obtain the differential equation

$$
\begin{aligned}
& \frac{\partial \hat{F}_{T}(\lambda, \beta)}{\partial \beta}+q[1-\beta(1-q) \hat{H}(\lambda)]^{-1} \hat{H}(\lambda) \hat{F}_{T}(\lambda, \beta) \\
= & -q \frac{\partial \hat{\tilde{H}}(\lambda, \beta)}{\partial \lambda} \hat{Q}_{T}(\lambda, \beta) .
\end{aligned}
$$

Using the explicit form given in Eq.(2), and defining the inverse operator, $\hat{Q}_{T}^{-1}(\lambda, \beta)$, in the usual way, we recast Eq.(6) in the form

$$
\hat{Q}_{T}(\lambda, \beta) \frac{\partial}{\partial \beta}\left[\hat{Q}_{T}^{-1}(\lambda, \beta) \hat{F}_{T}(\lambda, \beta)\right]=-q \frac{\partial \hat{\tilde{H}}(\lambda, \beta)}{\partial \lambda} \hat{Q}_{T}(\lambda, \beta) .
$$

From this expression the theorem I is established upon integration of both sides of Eq. $(7)$, because $\hat{Q}_{T}(\lambda, \beta=0)=1$ and $\hat{F}_{T}(\lambda, \beta=0)=0$.

The above Theorem reduces to the Wilcox theorem [1] when $q$ is set equal to unity.

This derivation is more direct than the one used in Ref.[1,2] for the Gibbsian ensemble. This is our central theorem.

From this, we deduce the following theorem by applying Theorem I using a similarity transformation to generate the $\lambda$-dependence of $\hat{H}(\lambda)$.

\section{THEOREM II:}

If $\hat{A}$ is an arbitrary operator, then the commutator $\left[\hat{A}, \hat{Q}_{T}(\lambda, \beta)\right]$ is given by

$$
\left[\hat{A}, \hat{Q}_{T}(\beta)\right]=q \hat{Q}_{T}(\beta) \int_{0}^{\beta} d u \hat{Q}_{T}^{-1}(u)[\hat{\mathcal{H}}, \hat{\bar{A}}(u)] \hat{Q}_{T}(u)
$$

where

$$
\begin{aligned}
& \hat{Q}_{T}(\beta)=[1-\beta(1-q) \hat{\mathcal{H}}]^{q /(1-q)}, \\
& \hat{\bar{A}}(u)=[1-u(1-q) \hat{\mathcal{H}}]^{-1} \hat{A}[1-u(1-q) \hat{\mathcal{H}}]^{-1} .
\end{aligned}
$$

Proof: We deduce this theorem by taking $\hat{H}(\lambda)$ in Theorem I as a similarity transformation of the following form

$$
\hat{H}(\lambda)=(\exp \lambda \hat{A}) \hat{\mathcal{H}}(\exp -\lambda \hat{A}) \text {. }
$$

Then it follows that

$$
\hat{Q}(\lambda, \beta)=(\exp \lambda \hat{A}) \hat{Q}_{T}(\beta)(\exp -\lambda \hat{A})
$$

where we used the definition in Eq.(8). Then, we obtain the following expressions that appear in Eq.(3)

$$
\begin{aligned}
& \frac{\partial \hat{Q}_{T}(\lambda)}{\partial \lambda}=\exp \lambda \hat{A}\left[\hat{A}, \hat{Q}_{T}(\beta)\right] \exp -\lambda \hat{A}, \\
& \text { and } \\
& \frac{\partial \hat{\tilde{H}}(\lambda)}{\partial \lambda}=\exp \lambda \hat{A}[\hat{\bar{A}}(u), \hat{\mathcal{H}}] \exp -\lambda \hat{A}
\end{aligned}
$$


which in Theorem I lead to the result in Eq.(8). In the limit when $q=1$, this goes to the well-known Kubo identity [2] which was of much use in his theory of irreversible processes.

We now employ Theorem I to deduce another important result concerning the parametric derivative of an Tsallisexpectation value of an arbitrary operator, often useful in computing the linear response function of a Tsallis-mean value of a quantity of interest. We must remark here that this definition of the mean value differs from the definition first proposed in [3] and has the advantage of having the property that the mean value of a scalar constant, $\mathrm{C}$, is $\mathrm{C}$ itself, which was not the case in its original formulation. While this entails some changes in the formalism it does not change the basic Legendre structure of the statistical mechanical principles. For a discussion of the implications and ramifications of these aspects, one may refer to [9]. For $q=1$ this goes over to the result for the usual thermodynamic average [2].

\section{THEOREM III:}

Define the Tsallis-expectation value of an arbitrary operator $\hat{B}[9]$ as

$$
\langle\hat{B}\rangle_{T}=\operatorname{Tr} \hat{B} \hat{Q}_{T}(\lambda, \beta) / \operatorname{Tr} \hat{Q}_{T}(\lambda, \beta)
$$

Then

$$
\begin{aligned}
\frac{\partial\langle\hat{B}\rangle_{T}}{\partial \lambda} & =-q \int_{0}^{\beta} d u\left\{\langle\hat{\mathcal{A}}(\lambda, u) \hat{B}\rangle_{T}-\langle\hat{\mathcal{A}}(\lambda, u)\rangle_{T}\langle\hat{B}\rangle_{T}\right\} \\
& =-q \int_{0}^{\beta} d u\left\{\langle(\Delta \hat{\mathcal{A}}(\lambda, y))(\Delta \hat{B})\rangle_{T}\right\}, \text { where } \\
\Delta \hat{A} & =\hat{A}-\langle\hat{A}\rangle, \mathcal{A}(\lambda, u)=\hat{Q}_{T}^{-1}(\lambda, u) \frac{\partial \hat{\tilde{H}}(\lambda, u)}{\partial \lambda} \hat{Q}_{T}(\lambda, u) .
\end{aligned}
$$

Proof: This follows upon differentiation of the defining expression in Eq.(12) and subsequently using Theorem I directly. Quite often, one uses this expression when $\hat{H}(\lambda)=\hat{H}_{0}+\lambda \hat{V}$, and evaluates the expression in Eq.(13) for $\lambda=0$ to obtain the correlation function of interest.

Finally, we develop a Karplus-Schwinger perturbation type theory for the Tsallis ensemble in the next Theorem. This theorem is not in the form of Theorem I but its proof involves steps similar to the one used in Theorem I and hence its inclusion here.

\section{THEOREM IV:}

If $\hat{H}=\hat{H}_{0}+\lambda \hat{H}_{1}$, then

$$
\begin{gathered}
\hat{Q}_{T}\left(\beta, \hat{H}_{0}+\lambda \hat{H}_{1}\right)=\hat{Q}_{T}^{(0)}\left(\beta, \hat{H}_{0}\right)-q \hat{Q}_{T}^{(0)}\left(\beta, \hat{H}_{0}\right) \cdot \lambda \int_{0}^{\beta} d u \\
\hat{Q}_{R}^{(0)-1}(\beta, \hat{H})\left(1-u(1-q)\left(\hat{H}_{0}+\lambda \hat{H}_{1}\right)\right)^{-1}\left(1-u(1-q)\left(\hat{H}_{0}\right)\right) \hat{\tilde{H}}_{1}(u) \hat{Q}_{T}\left(u, \hat{H}_{0}+\lambda \hat{H}_{1}\right) \\
\text { where } \hat{\tilde{H}}_{1}(u)=\left(1-u(1-q) \hat{H}_{0}\right)^{-1} \hat{H}_{1}\left(1-u(1-q) \hat{H}_{0}\right)^{-1} .
\end{gathered}
$$

Here

$$
\begin{aligned}
\hat{Q}_{T}\left(\beta, \hat{H}_{0}+\lambda \hat{H}_{1}\right) & =\left(1-\beta(1-q)\left(\hat{H}_{0}+\lambda \hat{H}_{1}\right)\right)^{q /(1-q)}, \\
\hat{Q}_{T}^{(0)}\left(\beta, \hat{H}_{0}\right) & =\left(1-\beta(1-q) \hat{H}_{0}\right)^{q /(1-q)} .
\end{aligned}
$$


Proof: Consider

$$
\begin{aligned}
\frac{\partial}{\partial \beta}\left\{\hat{Q}_{T}^{-1}\left(\beta, \hat{H}_{0}\right) \hat{Q}_{T}\left(\beta, \hat{H}_{0}+\lambda \hat{H}_{1}\right)\right\}= & \frac{\partial}{\partial \beta}\left\{\hat{Q}_{T}^{-1}\left(\beta, \hat{H}_{0}\right)\right\} \hat{Q}_{T}\left(\beta, \hat{H}_{0}+\lambda \hat{H}_{1}\right)+ \\
& \hat{Q}_{T}^{-1}\left(\beta, \hat{H}_{0}\right) \frac{\partial}{\partial \beta}\left\{\hat{Q}_{T}\left(\beta, \hat{H}_{0}+\lambda \hat{H}_{1}\right)\right\}
\end{aligned}
$$

Performing the indicated differentiations, and after some algebra we find

$$
\begin{aligned}
& \frac{\partial}{\partial \beta}\left\{Q_{T}^{-1}\left(\beta, \hat{H}_{0}\right) \hat{Q}_{T}\left(\beta, \hat{H}_{0}+\lambda \hat{H}_{1}\right)\right\}=-q \hat{Q}_{T}^{-1}\left(\beta, \hat{H}_{0}\right) \\
& \lambda\left(1-\beta(1-q)\left(\hat{H}_{0}+\lambda \hat{H}\right)\right)^{-1} \hat{H}_{1}\left(1-\beta(1-q) \hat{H}_{0}\right)^{-1} \hat{Q}_{T}\left(\beta, \hat{H}_{0}+\lambda \hat{H}_{1}\right)
\end{aligned}
$$

Introducing the notation given in Eq.(14), upon integration of both sides of Eq.(17) with respect to $\beta$, from 0 to $\beta$, and using $\hat{Q}_{T}(\beta=, 0 \hat{H})=1$, we obtain the result stated in Theorem IV above.

\section{Summary}

In summary, we have here deduced a set of four theorems on parametric differentiation of the operator defining the Tsallis ensemble, which we hope are of use in the same way as the Wilcox theorems were for the operator defining the Gibbsian ensemble.

Several versions of this paper were read by Professor Tsallis. Thanks are due to him for making valuable suggestions to improve the presentation. This work is supported in part by the Office of Naval Research.

\section{References}

[1] R. M. Wilcox, J. Math. Phys. 8, 962 (1967).
[2] W.T.Grandy, Jr. Foundations of Statistical Mechanics, Vol. I: Equilibrium Theory, and Vol. II:Nonequilibrium Phenomena, D. Reidel Publishing Co., Boston (1988).

[3] C. Tsallis, J. Stat. Phys. 52, 479 (1988). Since this paper first appeared, a large number of papers based on this work have been and continues to be published in a wide variety of topics. A list of this vast literature which is continually being updated and enlarged may be obtained from http://tsallis.cat.cbpf.br/biblio.htm

[4] A. K. Rajagopal, R. S. Mendes, and E. K. Lenzi, Phys. Rev. Lett. 80, 3907 (1998). See also an expanded version of this work by E. K. Lenzi, R. S. Mendes, and A. K. Rajagopal, Phys. Rev. E 59, 1398 (1999).

[5] E. T. Jaynes: Papers on Probabilty, Statistics, and Statistical Physics, edited by R. D. Rosenkrantz, D. Reidel Publishing Co., Boston (1983).

[6] M. L. Lyra and C. Tsallis, Phys. Rev. Lett. 80, 53 (1998).

[7] C. Anteneodo and C. Tsallis, Phys. Rev. Lett. 80, 5313 (1998).

[8] A. K. Rajagopal, Phys. Rev. Lett. 76, 3469 (1996).

[9] C. Tsallis, R. S. Mendes, and A. R. Plastino, Physica A 261, 534 (1998). 